\title{
CRYSTAL STRUCTURE OF ICE ACCRETED ON AN ICE SUBSTRATE*
}

\author{
By Olga B. Nasello, \\ (FAMAF, Universidad Nacional de Córdoba, 5000 Córdoba, Argentina) \\ LAURA LEVI, \\ (CEFA, Servicio Meteorológico Nacional, 1427 Buenos Aires, Argentina) \\ and FRANCO PRODI
}

(Laboratorio FISBAT - C.N.R., Reparto Nubi e Precipitazioni, 40126 Bologna, Italy)

\begin{abstract}
Thin ice deposits, grown on a prismatic ice substrate and the first layers of large deposits grown about a cylindrical ice collector, are studied. Experiments were performed at $30 \mathrm{~m} \mathrm{~s}^{-1}$ wind speed and $T_{\mathrm{a}}$ near $-20^{\circ} \mathrm{C}$. Progressive structural changes in thin accretions with time of water-injection were observed, when the substrate temperature was $T_{\mathrm{S}}>-5^{\circ} \mathrm{C}$. After $60 \mathrm{~s}$ injection, which determined a deposit $\sim 1 \mathrm{~mm}$ thick, the preferred orientation was established and the mean grain area was $\sim 0.01 \mathrm{~mm}^{2}$. Such structural changes did not appear along the initial layers of thick deposits. These differed mainly from the subsequent zone because of the larger value of the preferred angle between the $c$-axis and the growth direction. These effects are discussed by considering the nucleation and orientation selection processes which occurred during droplet freezing and the annealing effects that occurred below the growth front. The different $c$-axis orientations in the initial and main zones are related to the different $T_{\mathrm{S}}$ values during their formation.
\end{abstract}

\section{INTRODUCTION}

It is known that wind-tunnel ice accretions grown in a dry regime present typical $c$-axis orientations, which depend on the air and deposit temperatures, $T_{\mathrm{a}}$ and $T_{\mathrm{s}}$. A rapid examination of the accretion sections indicates that there is not a well-defined zone along which this characteristic orientation is established and that, even when the droplet collector is an ice cylinder, no relation may be observed between the accretion structure and that of the substrate cylinder. In order to explain this behaviour, Levi and Aufdermaur (1970) suggested a mechanism of orientation selection operating inside individual droplets, among icecrystal embryos nucleated on the substrate, so that crystals with unsuitable orientations would be immediately suppressed. On the contrary, Rye and Macklin (1973) considered that the selection process possibly occurred progressively among crystals with different orientations formed in adjacent droplets, and they suggested that a $0.5 \mathrm{~mm}$ thick accretion layer might be sufficient to attain a static regime by this mechanism. However, since no detailed analysis of such initial accretion layers has yet been performed, the different interpretations are only based on qualitative observations.

In order to obtain a better understanding of this phenomenon, in the present work the initial stage of the accretion process has been studied. With this purpose in mind, thin deposits formed by riming on a plane ice sub-

*See Annals of Glaciology, Vol. 6, 1985, p. 324 for extended abstract. strate and the initial layers of thick deposits grown around a bulk ice cylinder have been analysed.

\section{EXPERIMENTAL METHODS}

The experiments were performed in the vertical-icing wind tunnel of the FISBAT-C.N.R. Institute (Bologna, Italy). The arrangement used to grow cylindrical ice deposits has been described elsewhere (Levi and Prodi, 1978). In the present work, riming on a plane ice surface was achieved by replacing the rotating cylinder by an ice sheet, $1 \mathrm{~cm}$ $\times 1 \mathrm{~cm}$ in section, supported by a fixed metal holder. Thin accretions were grown using a spreading nozzle operating near the tunnel entrance, which provided droplets of $30 \mu \mathrm{m}$ mean volume diameter, at a rate of $\sim 800$ drops $/ \mathrm{mm}^{2} \mathrm{~s}$, with $25 \%$ maximum error. The wind speed was $30 \mathrm{~m} \mathrm{~s}^{-1}$. The sample holder could be warmed up electrically to obtain pre-established differences between $T_{\mathrm{a}}$ and $T_{\mathrm{S}}$. The sample temperature, measured by a platinum thermometer embedded in the ice, was recorded during the experiment. When droplets were injected, $T_{\mathrm{S}}$ could increase $2-3^{\circ} \mathrm{C}$ due to heat of melting released by droplet freezing. Thus, the mean value of $T_{\mathrm{S}}$, corresponding to each experiment, will be given in the following sections.

After each experiment, the sample was rapidly transferred to a cold room at $-10^{\circ} \mathrm{C}$ and a formvar replica of the treated surface was immediately obtained. These replicas revealed grain-boundary grooves and, when the grain dimensions were $>100 \mu \mathrm{m}$, they also showed thermal etch pits which could be used to determine the crystal orientation. In this case, histograms were obtained for the angle $\phi$ between the $c$-axes of new crystals and the normal to the surface.

Replicas of deposits grown in a dry regime about a cylindrical ice collector were used to analyse initial accretion zones. The cylindrical collector had a diameter of $1 \mathrm{~cm}$ and consisted of large ice crystals, most of them with their $c$-axes approximately tangential to the cylinder surface. The geometry of the grain presentation in the replica is different in this case. Since a cross-section of the cylinder is made, a circumference separates the grains of the polycrystalline substrate from the grains of the accreted deposit. In these conditions, the replicas give longitudinal sections of the crystals that are elongated in a radial direction, so that the grain width, $w$, can be compared with the crystal size in the case of thin accretions grown on a plane surface. Only the first $0.5 \mathrm{~mm}$ thick layer of the accreted deposits, corresponding to a growth time of about $30 \mathrm{~s}$, was investigated. During this time the deposit temperature, initially equal to $T_{\mathrm{a}}$, increased and approached its final value. This was reached after about $1 \mathrm{~min}$ of accretion. In the replicas, showing both grain boundaries and thermal etch pits (Aufdermaur and others, 1963), the grain-size and orientation were determined. 


\section{RESULTS}

\section{Riming on a prismatic ice surface}

The experiments were performed at values of $T_{\mathrm{a}}$ near $-20^{\circ} \mathrm{C}$ and at different values of $T_{\mathrm{s}}$. The analysis of 15 samples was carried out by determining the number and mean area of new crystals detected on the surface and, when possible, the $c$-axis orientation. Frequency distributions for the angle $\phi$ between the $c$-axes and the normal to the surface were derived from the analysis of no fewer than 100 grains. Typical results, for the fraction $H$ of the substrate surface covered by new crystals and for the mean grain area $\Sigma$, are given as a function of the drople injection time $t$ in Table I. It can be seen that an increase of $H$ with $t$ may be followed up to $t=10 \mathrm{~s}$, i.e. as long as the substrate was not completely covered by new crystals. At this stage, the accretion thickness was $\sim 15-20 \mu \mathrm{m}$. On the other hand, for $t<10 \mathrm{~s}$, crystals were rather small at all values of $T_{\mathrm{S}}$; when $T_{\mathrm{S}}>-5^{\circ} \mathrm{C}$, their area increased with $t$, reaching $0.01 \mathrm{~mm}^{2}$ for $t=60 \mathrm{~s}$, when the deposit thickness was about $1 \mathrm{~mm}$. However, the crystals did not increase in size with time at lower $T_{\mathrm{S}}$ (see lines 2 and 5 of Table I).

TABLE I. STRUCTURE OF THIN DEPOSITS GROWN ON A PRISM SUBSTRATE

$\begin{array}{rlrr}t & T_{\mathrm{a}} & T_{\mathrm{S}} & H \\ \mathrm{~s} & { }^{\circ} \mathrm{C} & { }^{\circ} \mathrm{C} & \% \\ 5 & -20 & -4 & 27 \\ 5 & -17.5 & -17 & 16 \\ 10 & -19.5 & -3 & 100 \\ 20 & -21 & -2 & 100 \\ 20 & -18 & -17 & 100 \\ 60 & -20.5 & -2 & 100\end{array}$

$\Sigma$

$\mu \mathrm{m}^{2}$

610

500

1600

3800

690

9450

The increase of $\sum$ with $t$, observed when $T_{\mathrm{S}}>-5^{\circ} \mathrm{C}$, was accompanied by a progressive change of the grain orientation. This is shown by the histograms in Figure la and b. The histogram for $t=15 \mathrm{~s}$ (Fig. 1a) has two maxima: one in the $75-90^{\circ}$ range, which corresponds to

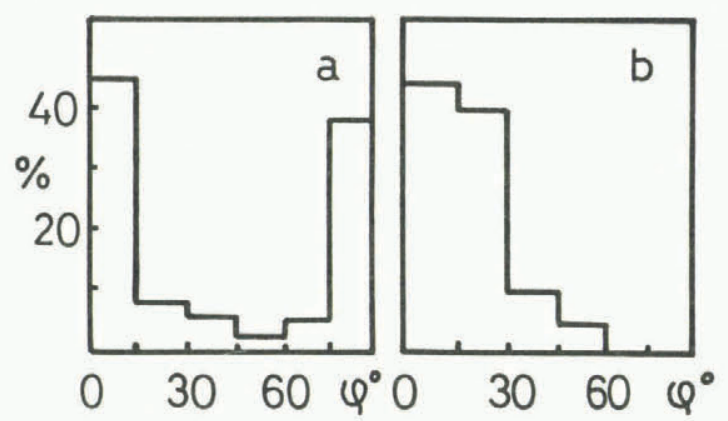

Fig. 1. Frequency distributions of $\phi$ in thin ice deposits grown on a prism substrate: (a) $T_{a}=-16^{\circ} \mathrm{C}$, $T_{s}=-4.5^{\circ} \mathrm{C}, \quad t=5 \mathrm{~s}$. (b) $T_{a}=-20.5^{\circ} \mathrm{C}, \stackrel{a}{T}_{s}=-2{ }^{\circ} \mathrm{C}$, $t=60 \mathrm{~s}$.

$c$-axes at a low angle to the substrate $c$-axis, the other in the $0-15^{\circ}$ range, which corresponds to the preferred $c$-axis orientation for ice accreted in a dry regime. At $t=60 \mathrm{~s}$ (Fig. 1b), the histogram shows that very few $c$-axes are oriented in the range $\phi>30^{\circ}$. In this case, the preferred $c$-axis orientation is clearly established.

\section{Cylindrical accretions}

Due to the elongated grain shape in accreted ice, measurements of the grain dimensions usually include the evaluation of the mean grain area $\bar{\sigma}$ and of the mean grain length and width, $\bar{l}$ and $\bar{w}$. Sometimes the dimensions $\bar{l}$ and $\bar{w}$ have been determined as an average of measurements performed separately for each grain. However, $\bar{w}$ has also been obtained from the length of a circumferential arc surrounding the accretion center, divided by the number of crystals this arc intersects.

In the present case, where the initial accretion structure had to be studied, $\bar{w}$ was obtained from the number of crystals intersecting an arc of the circular line which, in the replica, separates the initial bulk ice cylinder from the ice deposit. The initial grain area $\bar{\sigma}$, on the other hand, was determined by counting the crystals in the circular zone, about $0.5 \mathrm{~mm}$ wide, surrounding this line. The length $l$ was not measured but it can be evaluated approximately from $\bar{l}=\bar{\sigma} / \bar{w}$. This analysis was performed for five cylinders, in each case the studied zone containing about 100 grains. Examples of the results are given in Table II, where $\bar{w}$ and $\bar{\sigma}$ in columns 3 and 4 correspond to

TABLE II. STRUCTURE OF THE INITIAL AND MAIN ZONES OF THICK CYLINDRICAL ACCRETIONS

$\begin{array}{rrrrrr}T_{\mathrm{a}} & T_{\mathrm{S}} & \bar{w} & \bar{\sigma} & \bar{w}^{*} & \bar{\sigma}^{*} \\ { }^{\circ} \mathrm{C} & { }^{\circ} \mathrm{C} & \mu \mathrm{m} & \mu \mathrm{m}^{2} \times 10^{-3} & \mu \mathrm{m} & \mu \mathrm{m}^{2} \times 10^{-3} \\ -20 & -2 & 100 & 14 & 300 & 200 \\ -26 & -4 & 120 & 10 & 100 & 70 \\ -24 & -11 & 30 & 1 & 70 & 7\end{array}$

the first accretion layers analysed in the present work, while $\bar{w}^{*}$ and $\bar{\sigma}^{*}$ in columns 5 and 6 are from the results obtained by Levi and Prodi (1978) for the main zone. It can be seen that $\bar{w}$ is smaller than $\bar{w}^{*}$ but it has the same order of magnitude. On the other hand, $\bar{\sigma}$ is about one order of magnitude smaller than $\bar{\sigma}^{*}$, this difference being the consequence of the larger grain elongation in the main zone. Both $\bar{\sigma}, \bar{w}$, and $\bar{\sigma}^{*}, \bar{w}^{*}$ depend on the value of $T_{\mathrm{S}}$, given in column 2 of Table II. This can be seen by comparing lines 2 and 3 of the table, which correspond to samples grown at the same value of $T_{\mathrm{a}}$, but with $T_{\mathrm{S}}=-4^{\circ}$ and $-11^{\circ} \mathrm{C}$, respectively. The crystal dimensions are markedly smaller in the second case.

The values of $\bar{w}$ given in Table II may also be used to compare $\bar{w}^{2}$ with the area $\Sigma$ given in Table I. In fact, both $\bar{w}^{2}$ and $\Sigma$ represent the grain area normal to the droplet flux. It can be seen that, for $T_{\mathrm{S}} \geqslant-4^{\circ} \mathrm{C}, \bar{w}^{2}$ has the order of magnitude of $\Sigma$ at $t=60 \mathrm{~s}$. This signifies that the small crystals initially observed in the thin deposits (lines $1-5$ in Table I) may not be seen in the first layers of the thick deposits, when these have been kept, during the whole accretion time (usually $15-20 \mathrm{~min}$ ), at temperatures $T_{\mathrm{S}}$ only a few degrees below $0^{\circ} \mathrm{C}$.

The crystal orientation was also measured in the initial layers of some cylinders and this was compared with that found in the main zone. Due to the small crystal size in the initial zone, the etch pits used to determine the crystal orientation were not sufficiently well defined to determine the angle $\phi$. Thus, the angle $\eta$ between the radial direction and the $c$-axis projection on the section plane was measured for $\sim 100$ grains in each sample.

In Figure 2, typical examples are given of the $\eta$ histograms found for the initial and main zones, for deposits

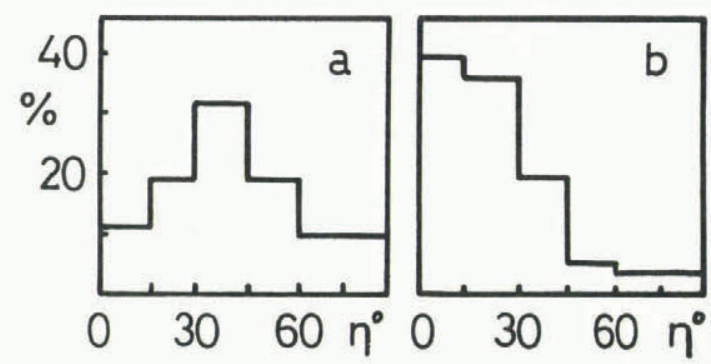

Fig. 2. Frequency distributions of $n$ in the initial (a) and main (b) zones of an ice deposit grown at $T_{a}=-22^{\circ} \mathrm{C}$, $T_{s}=-4^{\circ} \mathrm{C}$. 
grown at $T_{\mathrm{a}} \leqslant-20^{\circ} \mathrm{C}, T_{\mathrm{S}}>-5^{\circ} \mathrm{C}$. Notice that there is a preferred orientation in both histograms but the modal interval is located near $40^{\circ}$ in the first case, and near $10^{\circ}$ in the second. For the same values of $T_{\mathrm{a}}$ but $T_{\mathrm{S}}=-11^{\circ} \mathrm{C}$ (last line of Table II) the modal interval is located near $40^{\circ}$ in both histograms.

\section{DISCUSSION}

The contribution of different processes must be invoked, in order to give a satisfactory interpretation to the results given in the previous sections. These are:

(1) A process of nucleation that occurs in supercooled droplets when they spread on the substrate. This process would determine the formation of microscopic crystals with a mean cross-sectional area of $\leqslant 10^{3} \mu \mathrm{m}^{2}$, as shown in Table I for $t \leqslant 10 \mathrm{~s}$, and with a variety of different orientations, as shown in Figure 1 for $t=5 \mathrm{~s}$.

(2) A process of orientation selection, determined by the faster lateral growth of the crystals with a preferred orientation. This process would determine the progressive change of the rime structure with the injection time $t$, as shown in Table I and Figure 1 for $t$ increasing up to $60 \mathrm{~s}$.

(3) A process of grain growth that occurs in already formed ice layers while the accretion is in progress. This phenomenon is pronounced when the value of $T_{\mathrm{s}}$ is not much below $0^{\circ} \mathrm{C}$. It would be responsible for the differences in size observed between the crystals forming thin ice layers grown by riming on a prismatic substrate and those forming the initial layers of thick cylindrical accretions grown at $T_{\mathrm{S}}>-5^{\circ} \mathrm{C}$. Actually, only the latter deposits would suffer the suggested annealing effect.

Notice that a grain-size of $100-200 \mu \mathrm{m}$, observed in the initial layers of accretions grown at $-2^{\circ},-4^{\circ} \mathrm{C}$ (lines 1 and 2 in Table II) coincides with the dimensions which could be reached by grains of negligible initial size due to annealing at these temperatures during the $10-20 \mathrm{~min}$ accretion time (see, for instance, Azuma and Higashi, 1983). The effects of annealing would be less important in further accretion layers due to the shorter annealing time and the larger initial crystal size.

It is interesting to observe that, in each layer, the crystals reaching the largest sizes during freezing, i.e. those which could preferentially develop during annealing, would be those more conveniently oriented with respect to the accretion process. As a consequence, the crystal orientation prevailing in each accretion layer would be the typical one, corresponding to the mean temperature $T_{\mathrm{S}}$ that existed during growth of the layer. This fact would explain the difference between the histograms in Figure la and $b$. Actually, the first one would correspond to a mean value of $T_{\mathrm{S}}$, which is intermediate between that of the air and that prevailing during main growth. On the contrary, the latter value of $T_{\mathrm{S}}$ would be the one which determines the structure of the second histogram.

Finally, the location of the modal interval of the $\phi$ (or $\eta$ ) distributions, corresponding to larger angles in the initial zone rather than in the main zone of thick accretions, may also be considered as evidence that the preferred orientations in accreted ice and their variations with $T_{\mathrm{a}}, T_{\mathrm{S}}$ are intrinsically related to variations with the temperature of the growth mode of ice (Levi and Prodi, 1983). Actually, the presence of lobes, that could modify the position of this modal interval in thick knobbly accretions, may not operate in the initial layers (about $0.5 \mathrm{~mm}$ thick) grown directly on the smooth surface of the collector.

\section{REFERENCES}

Aufdermaur, A.N., and others. 1963. Kristallachsenlagen in Hagelkörnern, by A.N. Aufdermaur, R. List, W.C. Mayes, and M.R. de Quervain. Zeitschrift für Angewandte Mathematik und Physik, Bd. 14, Ht. 5, p. 574-89.

Azuma, N., and Higashi, A. 1983. Effects of hydrostatic pressure on the rate of grain growth in Antarctic polycrystalline ice. Journal of Physical Chemistry, Vol. 87, No. 21 , p. 4060-64.

Levi, L., and Aufdermaur, A.N. 1970. Crystallographic orientation and crystal size in cylindrical accretions of ice. Journal of the Atmospheric Sciences, Vol. 27, No. 3, p. 443-52.

Levi, L., and Prodi, F. 1978. Crystal size in ice grown by droplet accretion. Journal of the Atmospheric Sciences, Vol. 35 , No. 11 , p. 2181-84.

Levi, L., and Prodi, F. 1983. Effects of growth temperatures and surface roughness on crystal orientation of ice accreted in a dry regime. Journal of the Atmospheric Sciences, Vol. 40, No. 5, p. 1281-99.

Rye, P.J., and Macklin, w.C. 1973. Interpretation of crystallographic orientations in accreted ice. Journal of the Atmospheric Sciences, Vol. 30, No. 7, p. 1421-26. 